Prospective validation of a risk prediction model to identify high-risk patients for medication errors at hospital admission

Marieke M. Ebbens MSc $1,2,4^{\star}$,

Sylvia A. van Laar Bsc 1

Drs. Elsbeth J. Wesselink 3

Dr. Kim B. Gombert-Handoko 1

Prof. Dr. Patricia M.L.A van den Bemt 4

1 Department of Clinical Pharmacy and Toxicology, Leiden University Medical Center, Leiden, The Netherlands

2 Department of Pharmacy, St Jansdal Hospital, Harderwijk, The Netherlands

3 Department of Hospital Pharmacy, Zaans Medical Centre, Zaandam, The Netherlands

4 Department of Hospital Pharmacy, Erasmus University Medical Center, Rotterdam, The Netherlands

* Corresponding author: M.M. Ebbens Wethouder Jansenlaan 903844 DG Harderwijk The Netherlands

Telephone: +31 (0)341 463619 Fax: +31 (0)341 435534

E-mail: marieke.ebbens@gmail.com 


\title{
Prospective validation of a risk prediction model to identify high-risk patients for medication errors at hospital admission
}

\begin{abstract}
Background

Pharmacy led medication reconciliation in elective surgery patients is often performed at the preoperative screening (POS). Because of the time lag between POS and admission, changes in medication may lead to medication errors at admission (MEA). In a previous study, a risk prediction model for MEA was developed.

Objective

To validate this risk prediction model to identify patients at risk for MEA in a university hospital setting.

Methods
\end{abstract}

The risk prediction model was derived from a cohort of a Dutch general hospital and validated within a comparable cohort from a Dutch University Medical Centre. MEA were assessed by comparing the POS medication list with the reconciled medication list at hospital admission. This was considered the gold standard. For every patient a risk score using the risk prediction model was calculated and compared with the gold standard. The risk prediction model was assessed with receiver operating characteristic (ROC) analysis.

Results 
Of 368 included patients, 167 (45.4\%) had at least one MEA. ROC analysis revealed significant differences in the area under the curve of $0.535(\mathrm{p}=0.26)$ (validation cohort) versus $0.752(p<0.0001)$ (derivation cohort). The sensitivity in this validating cohort was $66 \%$ with a specificity of $40 \%$.

Conclusion and Relevance

The risk prediction model developed in a general hospital population is not suitable to identify patients at risk for MEA in a university hospital population. However, number of medications is a common risk factor in both patient populations and should thus form the basis of an adapted risk prediction model. 


\section{Introduction}

Medication errors at admission (MEA) occur frequently and have potential clinical relevance. ${ }^{1,2}$ To reduce these MEA, medication reconciliation implementation in hospital transitions is recommended by the World Health Organization and the Joint Commission..$^{3,4}$ The WHO describes medication reconciliation as "the formal process in which health care professionals' partner with patients to ensure accurate and complete medication information transfer at interfaces of care". ${ }^{3}$ Pharmacy-led medication reconciliation, which is considered the most cost-effective intervention ${ }^{5}$, can lead to a $66 \%$ reduction in medication errors. ${ }^{6}$ In the Dutch guideline on prevention of MEA, medication reconciliation is required within 24 hours of admission. ${ }^{7}$ In patients who will undergo elective surgery, medication reconciliation is often part of the preoperative screening (POS). ${ }^{8}$ However, the time window between the POS and admission is usually larger than 24 hours. ${ }^{9}$ Therefore, medication reconciliation should be repeated at admission to comply with the guideline.

To be able to select patients at high risk for MEA, risk factors need to be investigated. Hias et al. ${ }^{10}$ showed in a review that number of preadmission drugs and age have a predictive value for discrepancies. They advise to validate these variables in risk prediction models.

In agreement with the results of the above mentioned review, we identified the number of medications in an earlier study in a general hospital as a potential risk factor for discrepancies. In addition, respiratory comorbidity was identified as a potential risk factor for patients with MEA after medication reconciliation at the POS in a multivariate logistic 
model. Cardiovascular comorbidity was only significant in the univariate analysis. ${ }^{9}$ From the data of this study, a risk prediction model was constructed, containing these three variables. Although in this study the time between POS and admission was not identified as a potential risk factor, in $67 \%$ of the patients no difference in medication between POS and admission was found. Therefore, if patients at risk can be selected this could prevent a second interview in these patients. The ultimate aim is to use such a risk prediction model to identify patients at risk for MEA in all types of hospital settings, and to perform the second pharmacy-led medication reconciliation at admission in these patients at risk for an MEA only. This would result in a more efficient process, reducing workload for the clinical pharmacy and preventing redundant questions for patients at admission. To our knowledge there are no earlier studies that investigated a riskprediction model to select patients at high-risk for medication errors.

To validate if the risk prediction model developed in the general hospital patient population can predict medication errors at admission correctly in a university hospital population, we performed this study. The secondary objectives are to identify potential additional risk factors for MEA in a university medical patient population and to evaluate the characteristics of these MEA.

\section{Methods}

Study design 
For the derivation cohort we used an observational quantitative study design, in which medication discrepancies were identified by comparing the preoperatively screened medication with the medication at hospital admission.

For the validation cohort the same study design was used to retrieve the gold standard data. In addition, for each patient the outcome of the risk prediction model was calculated and compared to the gold standard.

\section{Derivation cohort}

The risk prediction model that was validated in this study was derived from a general hospital setting (Zaans Medical Centre, Zaandam, The Netherlands). The Zaans Medical Centre is a 300 bed general hospital with around 12,500 clinical admissions every year. Patients were included at the POS when given informed consent, were 18 years or older, and admitted to hospital for at least 24 hours. Exclusion criteria were patient or accompanying caregiver was not able to participate in the medication reconciliation, or a patient received additional pre-surgery medication reconciliation by the admitting medical ward. Patients were included between October 26 and December 18, 2015. The primary outcome was the proportion of patients with one or more MEA. ${ }^{9}$ Medication reconciliation consisted of a standardized medication interview with the patient performed by a researcher (pharmacist or pharmacist in training). The researcher was trained to work according to the medication reconciliation method used by the pharmacy technicians at the POS. When available, a recently obtained community pharmacy record, a medication list brought by the patient and/or his medication boxes were used to verify actual medication use with the patient. In case the patient could not answer 
these questions completely, the accompanying caregiver was interviewed as well. Medication reconciliation took place either pre- or post-operatively. Discrepancies between medications at the POS and medications at admission were divided into intentional and unintentional discrepancies using the patient medical record and if necessary information from the attending physician. Discrepancies were seen as intentional if home medication was intentionally altered during admission. Unintentional discrepancies are defined as home medication that was not correct in the electronic record and confirmed by a physician that it was unintentional. Unintentional medication discrepancies between the hospital electronic medical record and the actual used medication were defined as medication errors at admission (MEA). When an MEA was found, this was communicated with and corrected by the attending physician.

\section{Validation cohort}

The validation cohort was derived from patients admitted for elective surgery in a university hospital setting and performed at the Leiden University Medical Centre (LUMC) in The Netherlands. The LUMC is a 800 bed university hospital with around 25,000 clinical admissions every year. The study received a waiver from the Medical Ethics Committee of the LUMC, as it complied with the Medical Research in Humans Act. Patients were included between October 17, 2016 and August 29, 2017. The data was prospectively collected using the same methods as in the derivation cohort.

\section{Outcome}

The primary outcome was the proportion of patients with one or more MEA. This outcome was used as the gold standard, to which the outcome predicted by the risk 
prediction model was compared. Sensitivity and specificity, were reported. The area under the curve (AUC) of the receiving operating characteristics $(R O C)$ curves from the derivation cohort ${ }^{9}$ and the validation cohort were compared. Secondary outcomes were potential risk factors for MEA in the validation cohort, type and severity of the MEA as well as the type of medications involved in the MEA.

Candidate predictors

The following patient characteristics were collected in the validation cohort as candidate predictors of MEA: age, sex, time in days between preoperative screening and admission, number of medications at the POS and admission, level of education, presence of comorbidities (cardiovascular disease, hypertension, respiratory disease, cerebrovascular accident, kidney disorder, thrombosis/embolism, diabetes mellitus, thyroid disorder), ward type and medical specialty. In the medication reconciliation interview the number of medications and the level of education (primary school, secondary school, high school, vocational education, university of applied sciences or university) was determined.

Classification of medication errors and medication types

The MEA were classified by error type and severity. Three types of medication errors were defined: omission (not in the hospital record, but observed to be in use), commission (in the hospital record, but not observed to be in use) and frequency/dose (medication in the hospital record, but in another frequency or dose than observed). The severity of the MEA was classified using the NCC MERP medical error index (Figure 1). ${ }^{11}$ Category A and B were not included as medication errors in this study, because 
these are categories that did not reach the patient. Therefore MEA were classified in category $C$ to $I$. The identified MEA were assessed with respect to NCC MERP severity class by two hospital pharmacists (KG and ME) independently from each other. In case their assessments differed, they met to reach consensus. Anatomic Therapeutic Chemical (ATC) code of medications involved in MEA was noted, to evaluate which medication subclass was more involved in MEA. ${ }^{12}$

Risk prediction model

The risk prediction model, that was developed from the data of the derivation cohort ${ }^{9}$, was: Risk score $=0.152{ }^{*} \mathrm{~N}+0.907{ }^{*} \mathrm{C}+1.446{ }^{*} \mathrm{R}$. In which $\mathrm{N}=$ number of medications at POS, $\mathrm{C}=$ cardiovascular comorbidity, and $\mathrm{R}=$ respiratory comorbidity. Other patient characteristics did not remain significant in the multivariate risk prediction model. From the ROC curve of this development cohort the risk score of 0.5 was chosen as cut-off with a sensitivity of $70 \%$ and specificity of $62 \%$. For every patient from the validation cohort the risk score was calculated using this risk prediction model. Every patient with a risk score above 0.5 was defined as a patient at risk for MEA.

Model validation

The required sample size for the validation cohort was estimated at 162 patients with an MEA, using the sensitivity of $70 \%$, alpha of 0.025 and beta of 0.20 and a sensitivity of $60 \%$ or higher as equivalent proportion. Based on an estimated prevalence of $33 \%^{9}$, a sample size of 500 patients was calculated. The study protocol included an interim analysis after 300 patients to evaluate the prevalence of MEA and if necessary to adjust the sample size. The data were transferred from OpenClinica version 3.8 (OpenClinica 
LLC, Waltham, USA) to SPSS Statistics version 23 (IBM Corp, Armonk, NY) for analysis.

The proportion of patients with MEA was calculated dividing the patients with MEA by the total number of included patients. For every patient the actual occurrence of MEA was compared to the predicted occurrence of MEA. The diagnostic value of the prediction model was established by calculating the sensitivity and specificity. The ROC curves of the risk prediction model and these validation parameters were compared to establish the fit of the model.

Secondly, the association of all potential risk factors with the occurrence of MEA was analysed using univariate logistic regression. If the $p$-value was $<0.20$, the parameter was analysed in a stepwise backwards multivariate logistic regression model and was retained in the model if it changed the beta coefficient with more than $10 \%$. Odds ratio's (OR) and $95 \%$ confidence intervals $(95 \% \mathrm{Cl})$ were reported.

Type and severity of MEA and type of medication was analysed using descriptive statistics. 


\section{Results}

After screening of 1005 patients, 368 patients were included according to the study flow in Figure 2. The most common cause for exclusion was the availability of patients for the interview (59\%). Of these non-available patients 152 (24\%) were not included due to transfer to a medical ward where the study was not executed. Another $10 \%$ was due to the patient being asleep at the time the researcher arrived and $10 \%$ due to other medical staff that was with the patient.

Table 1 shows the general characteristics of the included patients of the validation cohort and derivation cohort. The mean age of the validation population was 61.3 years with a standard deviation (SD) of 13.6 compared to 61.8 with SD 16.3 in the derivation population. Men (52\%) and women (48\%) were included almost equally in the validation cohort, compared to $42 \%$ male and $58 \%$ female in the derivation cohort. On average a lag of 27.8 days existed between the POS and admission with a large variation (SD 31.6 days) compared to $32.8 \pm 24.8$ days in the derivation cohort. On average, patients used 4.7 medications (SD 4.2) at the POS in the validation cohort significantly more than the average of 3.7 medications (SD 3.5) in the derivation cohort. Furthermore the population of the derivation cohort significantly differed in level of education, and occurrence of cardiovascular disease, kidney disorder, and thrombosis/embolism.

Of the 368 patients included, 167 (45.4\%) had at least one MEA. For 8 patients the risk score could not be calculated because the cardiovascular comorbidity was unknown. For 360 patients the prediction of MEA using the risk prediction model is compared to the actual occurrence of MEA. This resulted in 108 patients with MEA that were correctly 
predicted to have MEA and 79 patient without MEA that were correctly predicted to having no MEA. 56 patients had MEA but it was predicted they would not have MEA and 117 patients had no MEA but were predicted to have MEA. The sensitivity in this validating cohort is $66 \%$, with a specificity of $40 \%$. The sensitivity of the derivation cohort is $70 \%$ with a specificity of $62 \%$. The ROC curves of the prediction model in the derivation and validation cohort are shown in figure 3 . The AUC of the validation cohort is $0.535(p=0.26)$. This means only half of the MEA is predicted correctly by this model and this is not significantly better than chance. The AUC of the ROC curve of the derivation cohort is $0.752(p<0.0001)$. This means about $75 \%$ of the MEA are correctly predicted by this model and this is significantly better than chance.

In Table 2 the results of the univariate logistic regression analysis are presented. Only the number of medications at admission OR $1.06(95 \% \mathrm{Cl} 1.01-1.12)$ was significantly associated with the occurrence of MEA. In the stepwise backwards multivariate logistic regression, the two potential risk factors that showed a p-value below 0.20 in the univariate analysis were analysed. None of the variables remained in the equation and therefore the results of the univariate analysis are the final results for the associated potential risk factors. No additional risk factors were identified.

In the 167 patients with at least one MEA, 302 MEA were found. Of these MEA 145 (48\%) were omissions, 90 (30\%) were commissions and 67 (22\%) consisted of changes in dose/frequency. $193(63 \%)$ of the MEA in 133 (36\%) patients, were classified to at least have the potential to be harmful (NCC MERP category D, E or F). Medications of the 'Alimentary tract and metabolism' (26\%) ATC class were most frequently involved in 
MEA, followed by 'Nervous system' (18\%), 'Dermatologic' (12\%) and 'Respiratory medication' (11\%).

\section{Discussion}

This study showed that the risk prediction model developed with data from the derivation cohort $^{9}$ is not suitable to predict MEA in patients in the validation cohort. In this study $45.4 \%$ of included patients had at least one MEA. This is substantially higher than the results of earlier studies ${ }^{6,13}$ and $12 \%$ higher than the derivation cohort ${ }^{9}$. This could be explained by the more complex patient population in the university hospital setting from the validation cohort. Cardiovascular comorbidity occurred almost twice as often $(21 \%$ versus $10 \%$ ) and kidney disorder and thrombosis occurred more than twice as often in the validation cohort (respectively $8 \%$ versus $3 \% ; 9 \%$ versus $2 \%$ ). Furthermore, more patients (36\% vs $9 \%)$ had a potentially harmful MEA in the validation cohort. This confirms the more complex patient population in the university hospital setting.

The higher percentage can also be explained by the higher number of medications used (average 4.7 compared to 3.7). In the logistic regression number of medications at the POS is significantly associated with the occurrence of MEA. This corresponds with the findings of earlier studies. 8,10

Compared to the derivation cohort more omissions were found, comparable with earlier studies. ${ }^{1,13}$ The ATC medication classes most frequently involved in MEA were the same as in the derivation cohort. The medication classes 'Alimentary tract and metabolism', 'Nervous system', 'Dermatologic', and 'Respiratory medication' should get more attention in the medication reconciliation process. Unfortunately the medication class is 
not suitable as a risk factor to predict MEA because most MEA are omissions and therefore the medication class is not known at the moment of risk assessment.

This is the first validation study of a risk prediction model for patients at risk for MEA. A strength of this study is that it included patients of all surgical specialties and therefore the findings are representative for daily clinical practice. The number of medications is represented in the risk prediction model and is an independent risk factor associated with MEA in the validation cohort as well, confirming the importance of this potential risk factor. Another strength of this study is that the derivation cohort and validation cohort are studied applying the same study procedures.

Some limitations need to be discussed. First of all more than half of the eligible patients are not included in the validation cohort. This is explained by the fact that patients who undergo surgery are often not in their hospital bed due to the surgery itself but also because of different appointments to help recover after surgery. However, we do not expect this to result in selection bias, as these logistic reasons apply to all patients. In February 2017 the gynaecology department of the LUMC introduced an intervention to perform a second medication reconciliation for every patient in the week before admission. Therefore when this intervention started the gynaecology department was excluded in this study. This means that only during half of the inclusion time of the validation cohort, patients of this specialty could participate in the study. However, we do not believe this affected the results because no difference has been demonstrated between the medical specialties. Another limitation of this study is that differences in medication overview after medication reconciliation can be caused by different approaches of the interview instead of actual changes in medication between the two 
medication reconciliation moments. Patients could forget to mention a medicine at the POS and remember it again at admission. We tried to minimize this risk by performing both medication reconciliation interviews according to the same protocol. However, we did not ask the patients when medication changes were discovered, where these changes originated from.

Future research is necessary to be able to identify high-risk patients with a better specificity and sensitivity. For the time being it is advised to perform a second medication reconciliation in elective patients at admission because $45 \%$ of patients still have MEA. To prevent the unnecessary performance of medication reconciliation in more than half of the patients better prediction models are necessary to select patients at high risk for an MEA. Future research that combines data from different patient populations would be helpful to establish this.

\section{Conclusion and Relevance}

The risk prediction model developed in a general hospital patient population is not suitable to identify patients at risk of medication errors at admission (MEA) in a university hospital patient population. This may be due to the more complex patients in the university hospital patient population. The number of medications is a common risk factor in both patient populations and should thus form the basis of an adapted risk prediction model. 


\section{References}

1. Tam VC, Knowles SR, Cornish PL, Fine N, Marchesano R, Etchells EE.

Frequency, type and clinical importance of medication history errors at admission to hospital: a systematic review. CMAJ, 2005;173(5):510-515. DOI:

10.1503/cmaj.045311

2. Quellenec B, Beretz L, Paya D, et al. Potential clinical impact of medication discrepancies at hospital admission. Eur J Intern Med, 2013;24:530-535. DOI: 10.1016/j.ejim.2013.02.007

3. World Health Organization. Assuring Medication Accuracy at Transitions in Care: Medication Reconciliation. http://www.who.int/patientsafety/implementation/solutions/high5s/h5s-sop.pdf Updated, September 2014. Accessed, April 2018.

4. Joint Commission Sentinel Event Alert - Using medication reconciliation to prevent errors. Issue 35; Jan 25, 2006

5. Karnon J, Campbell F, Czoski-Murray C. Model-based cost-effectiveness analysis of interventions aimed at preventing medication error at hospital admission (medication reconciliation). J Eval Clin Pract, 2009;15(2):299-306. DOI: $10.1111 / \mathrm{j} .1365-2753.2008 .01000 \cdot x$

6. Mekonnen AB, McLachlan AJ, Brien JA. Pharmacy-led medication reconciliation programmes at hospital transitions: a systematic review and meta-analysis. J Clin Pharm Ther, 2016;41(2):128-144. DOI: 10.1111/jcpt.12364 
7. Dutch Federatie Medisch Specialisten and Ministry of Health Welfare and Sports. Leidraad Overdracht van Medicatiegegevens in de keten. http://www.nhg.org/. Updated November 2017. Accessed December 30, 2017

8. van den Bemt PM, van den Broek S, van Nunen AK, Harbers JB, Lenderink AW, Medication reconciliation performed by pharmacy technicians at the time of preoperative screening. Ann Pharmacother, 2009;43(5):868-74. DOI:

10.1345/aph.1L579

9. Ebbens MM, Gombert-Handoko KB, Al-Dulaimy M, van den Bemt PMLA, Wesselink EJ. Risk factors for medication errors at admission in preoperatively screened patients. Pharmacoepidemiol Drug Saf. 2018 Mar;27(3):272-278. DOI: $10.1002 / p d s .4380$

10. Hias J, van der Linden L, Spriet I, et al. Predictors for unintentional medication reconciliation discrepancies in preadmission medication: a systematic review. Eur J Clin Pharmacol, 2017;73(11):1355-1377. DOI: 10.1007/s00228-017-2308-1

11. National Coordinating Council for Medication Error Reporting and Prevention (NCCMERP). Types of Medication Errors. 1996. http://www.nccmerp.org/typesmedication-errors. Updated 2001. Accessed April 2018.

12. WHO Collaborating Centre for Drug Statistics Methodology. Complete ATC index 2016. http://www.whocc.no/atcddd/. Updated December 20, 2017 Accessed December 30, 2017.

13. Kwan Y, Fernandes OA, Nagge JJ, et al., Pharmacist medication assessments in a surgical preadmission clinic. Arch Intern. Med, 2007;167(10):1034-1040. DOI: 10.1001/archinte.167.10.1034 
Figure 1 NCC MERP index for categorizing medication errors

\section{NCC MERP Index for Categorizing Medication Errors}
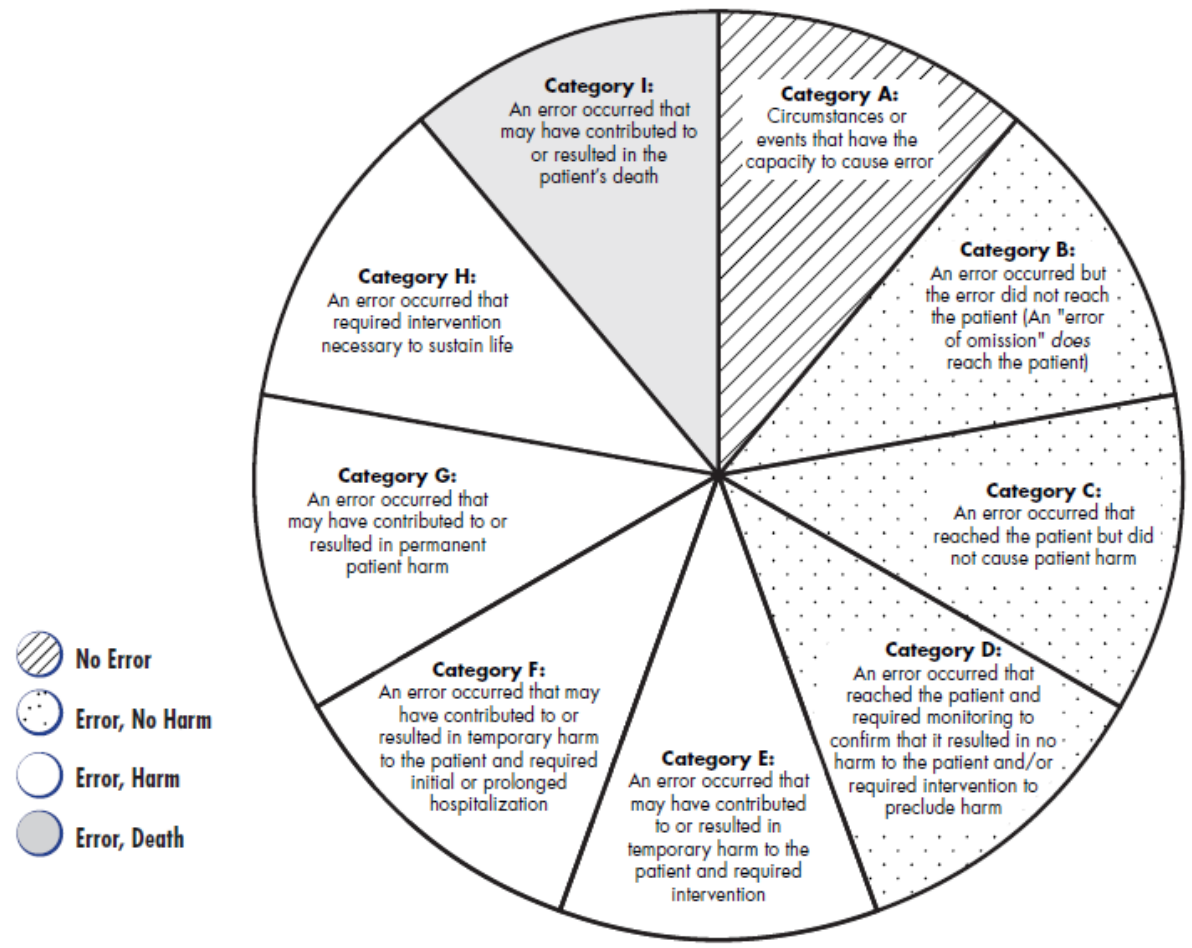

Definitions

Harm

Impairment of the

physical, emotional, or psychological function or structure of the body and/or pain resulting therefrom.

Monitoring

To observe or record relevant physiological or psychological signs.

Intervention May include change in therapy or active medical/surgical treatment.

Intervention

Necessary to Sustain Life

Indudes cardiovascular and respiratory support (e.g., CPR, defibrillation, intubation, etc.) 
Table 1 Patient characteristics for validation cohort $(n=368)$ and derivation cohort $(n=183)$

\begin{tabular}{|c|c|c|c|}
\hline & $\begin{array}{l}\text { Validation } \\
\text { cohort }\end{array}$ & $\begin{array}{l}\text { Derivation } \\
\text { cohort }\end{array}$ & p-value \\
\hline Age, mean \pm SD & $61.3 \pm 13.6$ & $61.8 \pm 16.3$ & 0.704 \\
\hline Sex (female), N (\%) & $176(48 \%)$ & $106(58 \%)$ & 0.199 \\
\hline $\begin{array}{l}\text { Days between POS and admission, } \\
\text { mean } \pm \text { SD }\end{array}$ & $27.8 \pm 31.6$ & $32.8 \pm 24.8$ & 0.062 \\
\hline $\begin{array}{l}\text { Number of medications at POS, mean } \pm \\
\text { SD }\end{array}$ & $4.7 \pm 4.2$ & $3.7 \pm 3.5$ & $0.006^{*}$ \\
\hline $\begin{array}{l}\text { Number of medications at admission, } \\
\text { mean } \pm \text { SD }\end{array}$ & $4.8 \pm 4.0$ & $3.6 \pm 3.5$ & $0.001^{*}$ \\
\hline 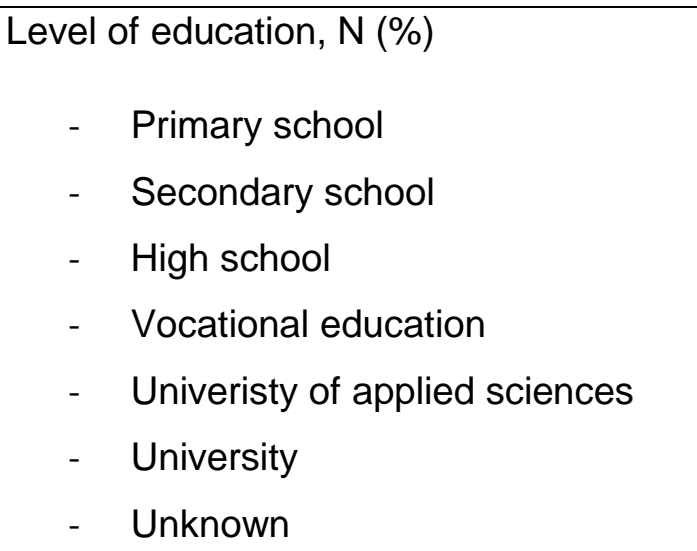 & $\begin{array}{l}35(10 \%) \\
72(20 \%) \\
25(7 \%) \\
98(27 \%) \\
69(19 \%) \\
43(12 \%) \\
26(7 \%)\end{array}$ & $\begin{array}{l}14(8 \%) \\
43(23 \%) \\
9(5 \%) \\
72(39 \%) \\
35(19 \%) \\
10(5 \%) \\
-\end{array}$ & $0.029^{*}$ \\
\hline \multicolumn{4}{|l|}{ Comorbidities, N (\%) } \\
\hline Cardiovascular disease & $75(21 \%)$ & $22(12 \%)$ & $0.012^{*}$ \\
\hline Hypertension & $136(37 \%)$ & $55(30 \%)$ & 0.104 \\
\hline Respiratory disease & $45(12 \%)$ & $22(12 \%)$ & 0.946 \\
\hline Cerebrovascular accident & $17(5 \%)$ & $10(5 \%)$ & 0.835 \\
\hline Kidney disorder & $30(8 \%)$ & $6(3 \%)$ & $0.020^{*}$ \\
\hline Thrombosis/embolism & $32(9 \%)$ & $4(2 \%)$ & $0.003^{*}$ \\
\hline Diabetes mellitus & $46(13 \%)$ & $19(10 \%)$ & 0.390 \\
\hline Thyroid disorder & $27(7 \%)$ & $17(9 \%)$ & 0.486 \\
\hline
\end{tabular}

Abbreviations: $\mathrm{POS}=$ preoperative screening, $\mathrm{SD}=$ standard deviation, ${ }^{*} \mathrm{p}<0.05$ 
Figure 3 Receiving Operater Characteristic curves of the prediction model in the derivation vs the validation cohort

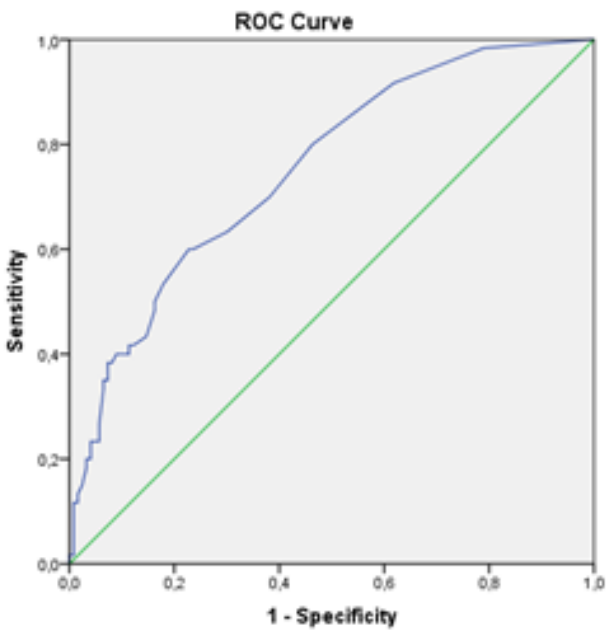

Diagonal segments are produced by ties.

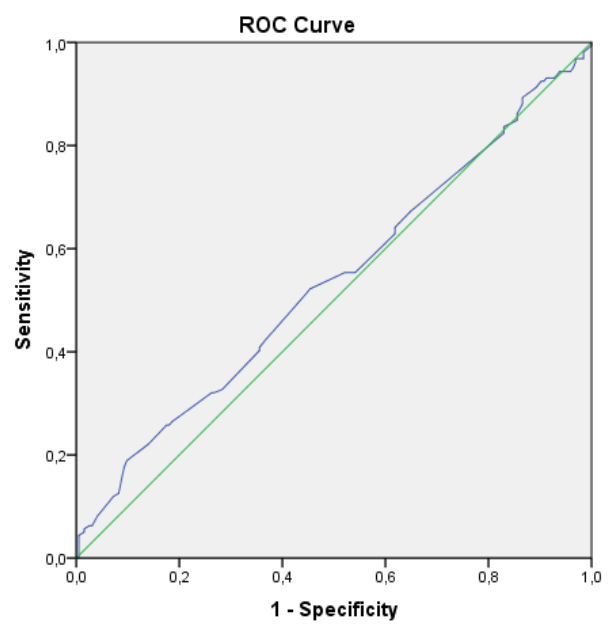

Diagonal segments are produced by ties.

Receiving Operator Characteristics curves; the curved line is the prediction of a medication error at admission (MEA) vs the real occurrence of MEA. The straight line is the reference. On the left: derivation cohort, the area under the curve (AUC) is $0.752 \mathrm{p}$-value <.0001. On the right: validation cohort, the AUC is $0.535 p$-value 0.26 . 
Table 2 Univariate logistic regression of patient factors associated with medication errors at admission

\begin{tabular}{|c|c|}
\hline Parameter & $\begin{array}{l}\text { Odds Ratio (95\%- } \\
\text { confidence interval) }\end{array}$ \\
\hline Age & $0.92(0.98-1.01)$ \\
\hline Sex & $0.92(0.61-1.39)$ \\
\hline Number of medications at preoperative screening & $1.06(1.01-1.12)^{*}$ \\
\hline $\begin{array}{l}\text { Time in days between preoperative screening and } \\
\text { admission }\end{array}$ & $1.00(0.99-1.01)$ \\
\hline Level of education ${ }^{1}$ & \\
\hline Secondary school & $1.59(0.70-3.60)$ \\
\hline High school & $2.14(0.70-6.97)$ \\
\hline Vocational education & $0.90(0.18-4.38)$ \\
\hline University of applied sciences & $1.03(0.47-2.27)$ \\
\hline University & $0.91(0.39-2.09)$ \\
\hline \multicolumn{2}{|l|}{ Comorbidities } \\
\hline Cardiovascular disease & $0.94(0.56-1.56)$ \\
\hline Hypertension & $1.07(0.70-1.63)$ \\
\hline Respiratory disease & $1.58(0.84-2.96)^{* *}$ \\
\hline Cerebrovascular accident (CVA) & $1.37(0.52-3.64)$ \\
\hline Kidney disorder & $1.23(0.58-2.61)$ \\
\hline Thrombosis/embolism & $1.59(0.77-3.31)$ \\
\hline Diabetes mellitus & $0.76(0.40-1.43)$ \\
\hline Thyroid disorder & $1.33(0.61-2.92)$ \\
\hline
\end{tabular}

${ }^{1}$ Primary school is the reference level

* $p$-value $<0.05$

${ }^{* *} p$-value $<0.20$ 